\title{
The relative effectiveness of stereometric and pattern stimuli in discrimination learning in children'
}

\author{
RHEA L, DORNBUSH ${ }^{2}$ ANO WILMA A, WINNICK \\ QUEENS COLLEGE OF THE CITY UNIVERSITY OF NEW YORK
}

The results of two discrimination experiments are presented. In the first, five-year olds learned size discriminations more rapidly with stereometric stimuli than with pattern stimuli; for seven-year olds there was little difference in learning the two discriminations. In Experiment II five-year olds learned size discriminations between perspective stimuli more rapidly than between control patterns without perspective. As in Experiment I both discriminations were equally easy for the seven-year olds. It was concluded that stimuli with depth provide added cues on which to base size discriminations.

The available evidence on the development of form perception based on discrimination situations indicates that the speed of learning is related to the type of stimulus used. Specifically, younger age groups form discriminations more readily with stereometric forms than with pattern forms (Alberts \& Ehrenfreund, 1951; Kuenne, 1946). This contrast between discriminations based on stereometric and pattern forms is also found in older age groups under some conditions. For example, in an investigation of the intermediate size problem Stevenson \& McBee (1958) found that six-year olds reached criterion with stereometric stimuli only.

Superior performance with stereometric stimuli would be expected to result from the greater number of cues associated with the depth characteristics of these objects. This analysis views differences in ease of discrimination among stimuli as related to the availability of useful cues.

Discrimination among pattern stimuli of younger children might be facilitated if depth cues were added to these stimuli without changing their two-dimensionality, that is, by adding internal lines giving the impresslon of depth. The two experiments in the present study manipulated the depth characteristics of the stimuli employed. The first experiment compared the abilities of five- and seven-year-old children to learn size discriminations with stereometric and pattern stimuli. Experiment II provided a test of the assertion that as depth cues become available discrimination learning will approach the level reached when stereometric stimuli are employed

Experimental Design. Experiments I and II both employed two age groups and two types of stimuli in an original learning and a 24-hr. transfer situation. Half of the Ss trained on one type of stimulus were presented with the other type in transfer; the remaining half were presented with the same stimulus as in original learning.

Subjects. Experiment I used 40 children, mean age 60-1/2 mo., and 40 children, mean age $85 \mathrm{mo}$.; Experiment II, 20 children, mean age 60-1/2 mo., and 20 children, mean age $84-1 / 2$ mo. Ss were drawn from N.Y.C. public elementary schools located in a middleclass neighborhood.

Apparatus and Materials. The stimuli were presented in a modified WGTA box. This apparatus measured $12-1 / 4$ in. in height, $17 \mathrm{in.} \mathrm{in} \mathrm{width,} \mathrm{and} 12 \mathrm{in}$. in depth. A Plexiglas window permitted viewing of stimuli. Two response buttons were centered below the window. Centered below these buttons was a shoot, from which marbles fell into a clear plastic receptacle resting on the table on which the box was placed.

Stimuli were presented on a milk platform inside the apparatus at the level of the window. Mlumination was provided by bulbs under the platform. A concealed Hunter timer recorded latencies. E's manipulations were shielded between trials.

The stereometric stimuli were of solid wood and covered with black construction paper. All other stimuli were made out of black construction paper. Where used, internal lines were drawn with white drawing pencil. All stimuli were attached to a white background 4 in. by 6 in. and were presented upright in the vertical plane.

The stereometric discrimination of Experiment I involved a 3 in. cube paired with a rectangle, 1-1/2 in. by 3 in. by 3 in. For the pattern task the stimuli were a 3 in. square, and a rectangle, 3 in. by 1-1/2 in. Perspective stimuli of the same dimensions as the pattern stimuli were used in Experiment II. Linear perspective was introduced into these drawings by internal lines. For the second discrimination the basic pattern figure was altered by arranging interior lines of similar extent without the cue of linear perspective.

Procedure. S was seated in front of the apparatus and familiarized with its use. After two pre-training trials the test stimuli were put in place. Half of the Ss were trained on one discrimination pair, the other half on the second discrimination pair. Positions of the stimuli were randomly varied in pre-arranged order. When $\mathrm{S}$ chose the positive stimulus a marble was automatically delivered into the clear plastic receptacle. Criterion was 10 consecutive correct responses. The following day $\mathrm{S}$ was brought to the testing room and told that he would play the same game. Criterion again was 10 consecutive responses. 
Table 1. Means and standard deviations for trials to reach criterion and latencies for size discriminations with stereometric and pattem stimuli

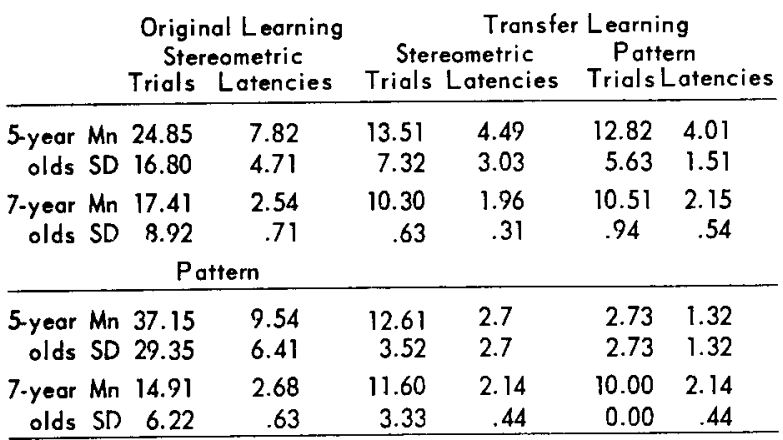

\section{Results}

Experiment I. Table 1 shows the means and SDs of trials to criterion and latencies (in seconds) for the two kinds of stimuli in the two age groups. Homogeneity of variance tests suggested " $t$ " comparisons between the two age groups and 2 by 2 analyses of variance with age groups for the transfer data. As a result of these analyses five-year olds were found to learn the stereometric size discrimination significantly more rapidly than the pattern size discrimination $(t=4.15, d f=38, p<.01)$.

Table 2. Means and standard deviations for trials to reach criterion and latencies for size discriminations with perspective stimuli and pattern analogues

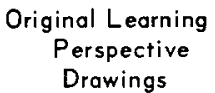

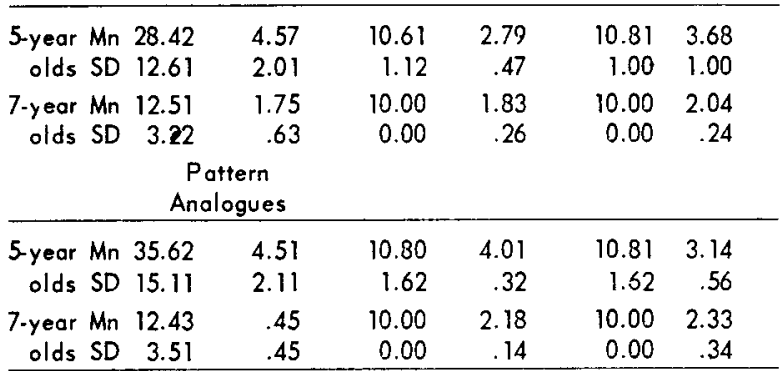

Experiment II. Table 2 shows the means and standard deviations of trials to criterion and latencies (in seconds) of perspective drawings and pattern analogues.

The original learning data indicated that the perspective size discrimination was learned more rapidly than the pattern analogue size discrimination $(t=1.2, d f=18$, $p<.075>.05$ ) for the five-year age group.

For seven-year olds the more difficult pattern analogue required increased time for the response. Further a significant interaction effect in mean latencies was found for the five-year age group $(F=5.6, d f=1 / 16$, $\mathrm{p}<.05)$. That is, when the original learning and transfer stimuli were not the same more time was needed for the responses in the transfer situation. Thus, a longer "decision" period on each trial was required rather than increased number of trials.

\section{Discussion}

Comparison provided in Experiment I between the discriminations based on stereometric and pattern stimuli were in accord with predictions based on previously published results (e.g., Stevenson \& McBee, 1958). That is, five-year-old children more readily learned a size discrimination with stereometric than with pattern stimuli while seven-year olds learned the discrimination with both types of stimuli with equal ease.

In Experiment II the size discrimination with linear perspective stimuli was learned more rapidly by fiveyear olds than the control size discrimination. The increased ease of discrimination accompanying the addition of the linear perspective cue to pattern stimuli strengthens the suggestion that the cues associated with three dimensional perception are important in making object stimuli more rapidly learned than pattern stimuli.

\section{References}

Alberts, E., \& Ehrenfreund, D. Transposition in children as a function of age. J. exp. Psychol., 1951, 41,30-38.

Kuenne, M. R. Experimental investigation of the relation of language to transposition behavior in young children. J. exp. Psychol., 1946, 36, 271-290.

Stevenson, H. W., \& McBee, G. The learning of object and pattern discriminations by children. J. comp. physiol. Psychol., 1958, $51,752-754$.

\section{Notes}

1. Supported by a National Institute of Mental Health pre-doctoral fellowship, (1-F1-MH-24, 181-01) to the first author.

2. Now at Douglass College, Rutgers-The State University. 